\title{
THE GLOBAL STABILITY OF A CLASS OF HISTORY-DEPENDENT MACROECONOMIC MODELS
}

\author{
Harbir Lamba ${ }^{1}$, Pavel KrejČí ${ }^{2,3}$ And Dmitrit RachinskiI ${ }^{4, *}$
}

\begin{abstract}
We consider piecewise-linear, discrete-time, macroeconomic models that have a continuum of feasible equilibrium states. The non-trivial equilibrium set and resulting path-dependence are induced by stickiness in either expectations or the response of the Central Bank. For a low-dimensional variant of the model with one representative agent, and also for a multi-agent model, we show that when exogenous noise is absent from the system the continuum of equilibrium states is the global attractor and each solution trajectory converges exponentially to one of the equilibria. Further, when a uniformly bounded noise is present, or the equilibrium states are destabilized by an imperfect Central Bank policy (or both), we estimate the size of the domain that attracts all the trajectories. The proofs are based on introducing a family of Lyapunov functions and, for the multi-agent model, deriving a formula for the inverse of the Prandtl-Ishlinskii operator acting in the space of discrete-time inputs and outputs.
\end{abstract}

Mathematics Subject Classification. 37N40, 93C55, 37B25.

Received January 24, 2019. Accepted November 30, 2019.

\section{INTRODUCTION}

Notions of friction and stickiness are widely accepted to exist in organizations, economies, and financial systems. Drawing intuition from mathematical physics, one can infer that a system with (dry) friction should have a continuum of equilibrium states. For example, an object can achieve an equilibrium on a curved surface at any point where the slope does not exceed the dry friction coefficient because the friction balances gravity.

There are various empirical regularities found in micro-, macro-, and organizational economic data, such as path-dependence, permanence, hysteresis, boom blessings and recession curses that are hard to replicate or explain with unique-equilibrium models. However, they can be accounted for quite naturally by the meta-stable states, with associated long timescale dynamics, that frictions at the micro-level introduce into such systems.

Based on this observation, in our first model we study how the introduction of internal frictions affects the dynamical properties of well-established single-equilibrium economic models. It is a fundamental economic question to determine what kinds of friction can actually arise from the behavior of economic agents and how

Keywords and phrases: Piecewise linear discrete time system, play operator, Dynamic Stochastic General Equilibrium model, multi-agent model, global stability.

1 Department of Mathematical Sciences George Mason University, VA, USA.

2 Institute of Mathematics of the Czech Academy of Sciences, Prague, Czech Republic.

3 Faculty of Civil Engineering, Czech Technical University, Prague, Czech Republic.

4 Department of Mathematical Sciences, The University of Texas at Dallas, TX, USA.

* Corresponding author: dmitry.rachinskiy@utdallas.edu

(C) The authors. Published by EDP Sciences, 2020 
these can be modeled. For example, some frictions may be a rational response to transaction costs while others may be caused by aspects of human psychology.

However friction can also be introduced at the macro-level via the action of a single large economic actor. In our second model we investigate this in the case of a Central Bank that operates a 'sticky' interest rate policy.

In this paper, we shall take a phenomenological approach to modeling frictions and use play operators which are common in physics and engineering applications. In an economic context, they are associated with notions of 'thresholds' and 'inaction bands'. These operators can be described in several equivalent ways, including piecewise linear (PWL) functions and via variational inequalities. We proceed by positing that both the current actual inflation rate and the current expectations of future inflation are related by a play operator (or by a combination of them in the multi-agent variant of the model) in a simple Dynamic Stochastic General Equilibrium (DSGE) macroeconomic model. Our analysis focuses on one particular feature of the adapted model with frictions. Namely, we consider local and global stability of this discrete-time PWL system, which naturally possesses a continuum of equilibrium states.

In the remainder of this section we provide some economic background and outline the structure of the paper.

Economic background and motivation. Seminal work by the likes of Walras and Jevons in the late 1800 's steered the field of economics away from the domain of philosophy, and laid the foundations for what they hoped would develop into a mathematically tractable (i.e. quantitatively predictive) scientific discipline. Early pioneers of what became known as Neoclassical Economics saw parallels between economic systems and the equilibrating forces in nature, and thus borrowed heavily from mathematical physics to derive both intuition and methodologies [42]. Despite concerns about the simplifying assumptions needed for this tâtonnement-driven view (e.g. $[28,41])$, the single-equilibrium approach is still considered good enough in many settings, and has not been supplanted by any widely-accepted, complete system. Significant developments have come from introducing concepts such as Rational Expectations and Sticky Models.

The former is the assumption of aggregate consistency in dynamic models [43] with the modeling assumptions. This view admits that agents' expectations about future uncertainties may be wrong individually, but in aggregate are in agreement with the model itself. In other words, although the future is not fully predictable, agents' expectations are assumed not to be systematically biased and to collectively use all relevant information in forming expectations of economic variables.

Meanwhile the latter includes the widely-used sticky models of Calvo [12] and the sticky-information of Mankiw and Reis [40]. These models are concerned with similar observations about the way in which real life agents do not instantaneously all move to the 'correct' price or opinion but rather do so at a fixed rate and can be represented mathematically by introducing a delay term into the relevant equations. In the absence of noise the same optimal equilibrium solution will be reached as if the stickiness were absent. Continua of possible equilibria can also occur in such models (see for example $[7,17]$ ) but usually in certain special cases (such as a passive interest-rate policy environment $[1,12]$ ) and are considered an extreme form of indeterminacy.

We note that there are other ways in which inaction can be justified as a rational response. The Rational Inattention literature (see for example $[53,54]$ ) treats economic agents as finite-capacity channels (in Information Theoretic terms) that can only (or choose to only) process new information at a finite rate. They then react optimally in the presence of these constraints but this can involve long delays and periods of non-reaction to changing economic circumstances (see [44] for a DSGE model with rational inattention).

There are also Instantaneous Control Models [55] where a (costly) control mechanism ensures that some quantity never leaves a specified interval by kicking in at the boundaries of the interval. For example, the inventory in a warehouse might be regulated by selling the surplus at a discount when it is full and having to buy at a premium when it is empty. A play operator can indeed fit into this framework but we shall focus upon dynamic rather than stochastic properties.

A robust empirical feature of economic output for developed countries is that output and output growth are non-normally distributed, exhibiting fat tails and excess kurtosis. In many models such booms and busts are explained by the occurrence of large (unpredictable) exogenous shocks followed by tranquil periods when nothing leads to non-normality. Explaining such regularities within linear unique-equilibrium economic models 
often involves adding a posteriori assumptions such as the existence of an eigenvalue with largest modulus close to, but inside, the unit circle (the Unit Root Hypothesis). A better model should generate non-normality of the output data from within the theory.

The critique of unique-equilibrium models has a long history which we shall not attempt to detail here. For example, many have eloquently pointed out fundamental issues with the assumed equilibrating processes and the ways in which the "aggregation problem" was being solved (e.g. [14, 27, 48, 52]). This approach is taken by multi-agent models including models of agents' behavior under imperfect information, cognitive limitations, or endogenously generated "animal spirits" [19]. Here, the themes of confusion, rational inattention, simplification, and bounded rationality take center stage $(e . g .[18,54])$. Non-normality in these models can be associated with a lack of independence between agents' actions that violates a key condition of the Central Limit Theorem. Most of these models are however purely numerical, and incorporate assumptions inspired by the social sciences (e.g. psychology and sociology) in order to add more realistic behavior to the modeling construct of individual actors (much recent work has focused on the question of whether individual actors can ever be sufficiently rational).

Our approach is complementary to many of the above-referenced views. In this paper, we argue that the lingering constraint of a single equilibrium in many such models is unnecessary. Hysteresis is a well-understood property that describes the "stickiness" observed in many physical systems (e.g. plasticity, magnetism), and which explains how multiple stable equillibria can arise. Hysteresis has only recently been explicitly considered in economic time series such as the unemployment rate $[5,6,16]$. The form of stickiness that we use is, to our knowledge, new in a macroeconomic setting and differs from, for example, the stickiness of the Calvo pricing model [12] where hypothetical agents are only allowed to adjust (to the correct price) at a fixed rate. The way in which we incorporate stickiness into the model will be justified and described more fully below but, briefly, our sticky variables can only be in one of two modes. They are either currently 'stuck' at some value or they are being 'dragged' along by some other (related) variable because the maximum allowable difference between them has been reached. Hence, our agents are truly stuck (not just delayed) until forced to adjust by some discrepancy. If an equilibrium is reached it is determined by the prior states of the system, and a continuum of feasible equilibria is an intrinsic feature of the model (note that other forms of discontinuity, unrelated to stickiness or the play operator, have also been found to induce history dependence [24]).

Real-world economic actions are rarely predictable or reproducible enough for a single model to be definitively 'accurate' and we believe there is much to be gained by studying phenomenological models that focus on particular qualitative features. For example, the empirical research into how inflation expectations are formed is extensive but far from conclusive, see [10, 11, 15, 45, 49]. However the idea of threshold effects and a "harmless interval' of inflation is not new to economics $[8,22,29,31,57]$ and is naturally captured using a play operator. A population may concurrently be subject to many human limitations (e.g. rational inattention and bounded rationality and confusion, etc.), but for our purposes it is enough to assume that, when aggregated, individual actors behave according to the play operator we describe, and we remain relatively agnostic as to which mechanism may be driving the general features of the play operator.

We also believe that introducing new, plausible, classes of perturbations to standard models can provide valuable insights into the robustness of the standard solutions to changes in the modeling assumptions [23], especially if they are amenable to mathematical analysis. Play operators provide such a class.

In a sense, the object of study of this paper is not an actual economy but instead the class of standard unique-equilibrium DSGE models that are supposed to mimic actual economies. This means that we can leave the defense of many of the DSGE modeling assumptions to economists (indeed we hope that work such as this will help undermine some of those assumptions). For example, the rationale behind the Taylor Rule is shaky at best but it has the benefit of fitting easily within a standard mathematical framework. Our insertion of a play operator into the Taylor Rule, and its rational interpretation, is a plausible mechanism for adding stickiness into the interest rate setting mechanism whose effect on stability etc can be investigated analytically.

We take a similar position on the questions of how to measure the various system parameters. For a dynamical systems study, where we attempt to identify basic properties of the phase and parameter spaces, we regard such considerations as secondary. 
Structure of the paper. In the next section, we present a discrete-time 3-dimensional PWL macroeconomic model with sticky inflation expectations modeled by a play operator. The model uses the notion of a representative agent. When the exogenous noise terms are absent from the system, it has a line segment of equilibrium points. Section 3 contains the main results. In particular, in the system without exogenous noise, a simple condition ensures that the line segment of equilibrium points is the global attractor (Sect. 3.1). In the presence of uniformly bounded noise we obtain an estimate of the globally attracting domain which is proportional to the supremum norm of the noise. Interestingly, this estimate is uniform with respect to the parameter that controls the amount of stickiness in the expectation of future inflation rate. We then consider further variants of the model. First, we add stickiness into the response of the Central Bank (Sect. 3.2). This can destabilize the equilibrium states (leading to periodic, quasiperiodic or more complex dynamics [3] with associated border collision bifurcations that are typical of piecewise smooth systems [4]) but the system still possesses a bounded globally attracting domain. Then we consider a multi-agent variant of the model (Sect. 3.3). This is an $(n+2)-$ dimensional PWL system with $2 n$ switching surfaces. We show that the $n$-dimensional continuum of equilibrium states of this system is the global attractor and each solution trajectory converges exponentially to one of the equilibria. Finally, the proofs based on constructing a family of Lyapunov functions are presented in Section 4. In order to apply the Lyapunov function to the multi-agent model, we adapt a technique from the theory of hysteresis operators $[9,30]$. Namely, an explicit formula for the inverse of the Prandtl-Ishlinskii operator acting in the space of discrete time inputs and outputs is derived and used. We conclude with a summary of the main results and some suggestions for future work.

\section{THE MODEL}

\subsection{DSGE modeling framework}

The standard approach to the problem of aggregating expectations is to introduce a 'Representative Agent' whose expectations are fully-informed and rational and consistent with the model itself. Here, an aggregation of boundedly rational agents into a similar Representative is required. Our approach is similar in spirit to that of De Grauwe [19] but we use a different model of boundedly rational agents' behavior.

We start from a toy dynamic stochastic general equilibrium (DSGE) macroeconomics model, which includes aggregate demand and aggregate supply equations

$$
\begin{aligned}
& x_{t}=b_{1} p_{t}+\left(1-b_{1}\right) x_{t-1}+b_{2} y_{t}+\eta_{t}, \\
& y_{t}=\left(1-a_{1}\right) y_{t-1}-a_{2}\left(r_{t}-p_{t}\right)+\epsilon_{t},
\end{aligned}
$$

augmented with the interest rate-setting Taylor rule

$$
r_{t}=c_{1} x_{t}+c_{2} y_{t}+\xi_{t}
$$

where $y_{t}$ is the employment rate (or output gap), $x_{t}$ is the inflation rate, $r_{t}$ is the interest rate, $p_{t}$ is the economic agents' aggregate expectation of the future inflation rate, $\eta_{t}, \epsilon_{t}, \xi_{t}$ are exogenous noise terms, and $t \in \mathbb{N}$. The parameters satisfy

$$
0 \leq a_{1}<1, \quad 0<b_{1}<1, \quad a_{2}, b_{2}, c_{1}, c_{2}>0
$$

The variables are all relative to reference values so that the set of equilibria in the noise-free system forms a line segment passing through the origin. When the noise-free system (2.1)-(2.2) is closed using Rational Expectations to define $p_{t}$ the equilibrium is unstable if $c_{1}<1$. Intuitively, this is due to runaway inflation caused by an under-responsive Central Bank and occurs in our variant models. Thus we also enforce $c_{1}>1$.

This model (and the parameters used) is close to the model used in [19] but simpler in that we do not include the aggregate expectation of the employment rate and the correlation between the subsequent values of the 
interest rate. The inclusion of such factors does not affect our most significant qualitative observations, but would complicate some aspects of the rigorous analysis that we present. ${ }^{1}$

The novelty of our modeling strategy is in how we define the relationship between the aggregate expectation of inflation $p_{t}$ and the inflation rate $x_{t}$.

\subsection{Sticky expectations of inflation}

We start from the empirical evidence cited above that an individual agent's expectations are often sticky and may lag behind the currently observable values before they start to move. We also posit that this gap between future expectations and current reality cannot grow too large. We then imbue our now boundedly rational Representative Agent with these same properties. More precisely, we assume the following rules that define the variations of the expectation of future inflation rate $p_{t}$ with the actual inflation rate $x_{t}$ at integer times $t$ :

(i) The value of the difference $\left|p_{t}-x_{t}\right|$ never exceeds a certain bound $\rho$;

(ii) As long as the above restriction is satisfied, the expectation does not change, i.e. $\left|x_{t}-p_{t-1}\right| \leq \rho$ implies $p_{t}=p_{t-1}$

(iii) If the expectation has to change, it makes the minimal increment consistent with constraint (i).

Rule (ii) introduces stickiness into the dependence of $p_{t}$ on $x_{t}$, while (i) states that the expected inflation rate cannot deviate from the actual rate more than prescribed by a threshold value $\rho$. Hence $p_{t}$ follows $x_{t}$ reasonably closely but on the other hand is conservative because it remains indifferent to variations of $x_{t}$ limited to a (moving) window $p-\rho \leq x \leq p+\rho$. The last rule (iii) enforces continuity of the relationship between $p_{t}$ and $x_{t}$ and, in this sense, can be considered as a technical modeling assumption that is mathematically convenient.

Rules (i)-(iii) are expressed by the formula

$$
p_{t}=x_{t}+\Phi_{\rho}\left(p_{t-1}-x_{t}\right), \quad t \in \mathbb{N}
$$

with the piecewise linear saturation function

$$
\Phi_{\rho}(v)=\left\{\begin{array}{rcc}
\rho & \text { if } & v \geq \rho, \\
v & \text { if } & -\rho<v<\rho \\
-\rho & \text { if } & v \leq-\rho .
\end{array}\right.
$$

Equations (2.1) and (2.2), completed with formulas (2.3) and (2.4), form a closed 3-dimensional PWL model for the evolution of the aggregated variables $x_{t}, y_{t}, p_{t}$. Another variant of this model has been considered in [3]. Iterations of the system (2.1)-(2.4) must start from a set of initial conditions $x_{0}, y_{0}, p_{0}$ satisfying $\left|x_{0}-p_{0}\right| \leq \rho$.

Some further terminology will be useful. Denote by $\mathcal{S}$ the set of all real sequences $x=\left(x_{0}, x_{1}, \ldots\right)$. For a given parameter $\rho>0$, the play operator $\mathfrak{p}_{\rho}:[-\rho, \rho] \times \mathcal{S} \rightarrow \mathcal{S}$ is defined as the mapping which with a given initial condition $s_{0} \in[-\rho, \rho]$ and a sequence $x \in \mathcal{S}$ associates the sequence

$$
p=\left(p_{0}, p_{1}, \ldots\right)=\left\{\mathfrak{p}_{\rho}\left[s_{0}, x\right]_{t} ; t \in \mathbb{N} \cup\{0\}\right\} \in \mathcal{S}
$$

according to the formula (2.3) with $p_{0}=x_{0}-s_{0}$ [30]. The parameter $\rho$ is called the threshold, see Figure 1 (left). A dual mapping $\mathfrak{s}_{\rho}:[-\rho, \rho] \times \mathcal{S} \rightarrow \mathcal{S}$, which is defined by the relationship

$$
s_{t}=\Phi_{\rho}\left(x_{t}-x_{t-1}+s_{t-1}\right), \quad t \in \mathbb{N}
$$

\footnotetext{
${ }^{1}$ More complicated variants of DSGE models, employing many more variables to represent different sectors of the economy, are widely used by central banks to help determine interest rate policy $[13,25,51,56]$. The DYNARE software platform supporting both DSGE models relying on the rational expectations hypothesis and some models where agents have limited rationality or imperfect knowledge of the state of the economy is available at http://www.dynare.org/.
} 

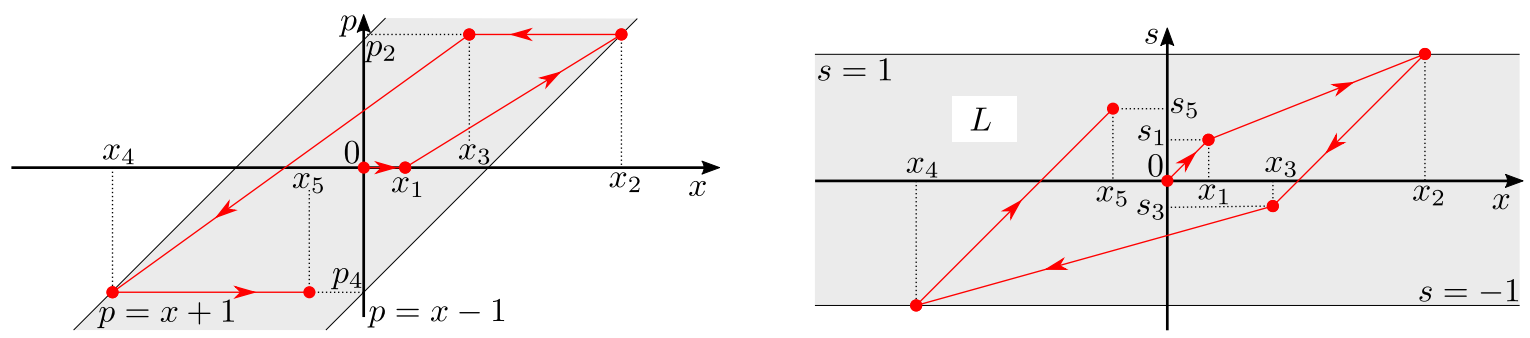

FiguRE 1. Graphical representation of the play and stop operators with the threshold $\rho=1$. Left: Example of an input-output sequence $\left(x_{t}, p_{t}\right)$ of the play operator. For each $t,\left(x_{t}, p_{t}\right)$ is the nearest point to $\left(x_{t-1}, p_{t-1}\right)$, which belongs to the band $|x-p| \leq 1$ (shown in grey) and has $x=x_{t}$. Right: The corresponding input-output sequence $\left(x_{t}, s_{t}\right)=\left(x_{t}, x_{t}-p_{t}\right)$ of the stop operator satisfies $\left|s_{t}\right| \leq 1$ for all $t$.
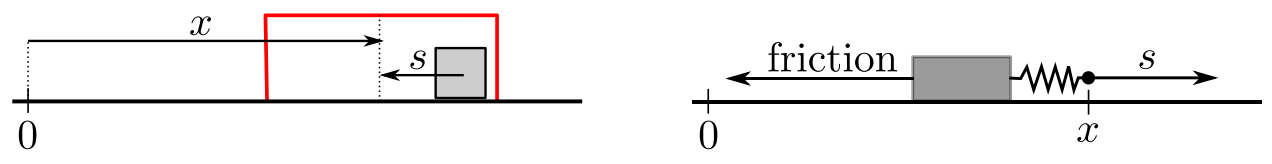

Figure 2. Continuous time mechanical interpretations of the stop operator. Left: The position $x$ of the center of the frame, which has length $2 \rho$, is the input; the relative position $s$ of the center of the frame with respect to the center of the grey box is the output. The frame moves and drags the box creating the input-output sequence $(x, s)$. Right: Schematic of the Prandtl's model of quasi-static elastoplasticity [46]. The grey box is not moving unless the absolute value of the force $s$ of the ideal spring reaches the maximal value $\rho$ of the Coulomb friction force between the box and the surface (equivalently, $s$ can denote the elongation of the spring); $x$ is the coordinate of the end of the spring.

for an arbitrary pair $\left(s_{0}, x\right) \in[-\rho, \rho] \times \mathcal{S}$, is known as the stop operator

$$
s=\left(s_{0}, s_{1}, \ldots\right)=\left\{\mathfrak{s}_{\rho}\left[s_{0}, x\right]_{t} ; t \in \mathbb{N} \cup\{0\}\right\} \in \mathcal{S},
$$

see Figure 1 (right). By definition the play and stop operators sum up to the identity:

$$
p_{t}+s_{t}=x_{t}
$$

for the sequences $(2.5),(2.7)$.

One can think of the play operator as having two modes, see Figure 2 (left). A 'stuck mode' where it will not respond to small changes in the input $x_{t}$ and a 'dragged mode' where the absolute difference between the input $x_{t}$ and output $p_{t}$ are at the maximum allowable and changes to the input, in the correct direction, will drag the output along with it. Further, in the context of our model, the output of the stop operator $s_{t}$ measures the difference between the inflation rate and the expectation of the future inflation rate, hence $s_{t}$ remains within the bound $\left|s_{t}\right| \leq \rho$ at all times. We shall refer to the variable $s_{t}=x_{t}-p_{t}$ as the perception gap. Interestingly the explicit relationship (2.6) has been observed in actual economic data [20,21]. Figure 2 (right) gives an interpretation of the stop operator combing an ideal spring and a dry friction element as used in mechanics.

\subsubsection{The aggregation problem}

The standard approach to the problem of aggregating expectations is to introduce a Representative Agent whose expectations are fully-informed, rational and consistent with the model itself. Here, even if we suppose that 
each agent acts like a play operator, then an aggregation of boundedly rational agents into a single Representative is required.

The aggregate of even just two play operators with differing thresholds is no longer a play operator - although the output will still display inactive (as well as less active) modes and there is still a maximum allowable difference between the input and output. However play (and stop) operators are just special cases of a wider class of Prandtl-Ishlinskii or PI operators.

PI operators have a remarkable aggregation/composition property. When connected together in an arbitrary network (under mild technical conditions) they collectively act as a single, but different, PI operator. Thus, as long as individual agents are represented by PI operators, there is a rigorous aggregation process by which a network of interacting heterogeneous agents can be reduced to a single Representative Agent. This ability to rigorously aggregate non-trivial agents is very unusual - and not just within economics. The result and further references to PI operators can be found in [32]. Furthermore this new composite PI operator can be identified analytically in simple cases or, in general, by measuring the network's response to a monotonic input.

So let us return to the expectations aggregation problem. We can imagine the agents in the economy/model being connected in a way that reflects how much influence expectations (or, say, wage increases) affect their neighbors. Then each agent's individual inflation expectation is modeled by a play operator (or by something 'close' within the space of PI operators) whose input is some combination of both the actual inflation rate and the expectations of their neighbors. By the composition property of PI operators, the aggregate response will also be a (probably quite complicated) PI operator that should nevertheless still have stuck, less active, and more active regimes while limiting the difference between the actual and aggregated expected inflation.

Importantly, when agents are connected into a network structure, internal 'cascades' of changes in expectations can occur. For example, if one especially significant agent suddenly starts to increase their expectation of inflation, this may trigger increases in its network neighbors' expectations and so on. Nevertheless the possibility of such cascades is still captured by the composite PI operator - if cascades can occur then the output of the operator when given a smoothly varying input has discontinuities that correspond to the cascades (see [32]).

Rather than study directly a sticky expectations model with a single, complicated, aggregate PI operator we start instead by assuming that the aggregated PI function is itself just a single play operator - this case can be analyzed in detail and is a necessary first step for a deeper understanding of the dynamics of such systems. The result for a multi-agent model is stated in Section 3.3 and proved in Section 4.3. Also, the space of all possible boundedly rational perturbations to rational models is very large and very hard to study rigorously or even define. This makes the analysis of tractable, plausible, boundedly-rational variants of rational models of independent interest and provides an additional justification for our non-standard but conceptually simple Representative Agent.

\section{MAin RESUlts}

\subsection{Autonomous system}

We first consider system (2.1)-(2.3) without noise terms:

$$
\begin{aligned}
& x_{t}=b_{1} p_{t}+\left(1-b_{1}\right) x_{t-1}+b_{2} y_{t}, \\
& y_{t}=\left(1-a_{1}\right) y_{t-1}-a_{2}\left(c_{1} x_{t}+c_{2} y_{t}-p_{t}\right), \\
& p_{t}=x_{t}+\Phi_{\rho}\left(p_{t-1}-x_{t}\right) .
\end{aligned}
$$

Equilibrium points of this system form a line segment

$$
\mathfrak{A}=\left\{\left(x_{*}, y_{*}, p_{*}\right)(u): x_{*}(u)=\kappa u, y_{*}(u)=\frac{b_{1}}{b_{2}} u, p_{*}(u)=(\kappa-1) u,|u| \leq \rho\right\}
$$


with

$$
\kappa=\frac{a_{2}\left(b_{2}+b_{1} c_{2}\right)+a_{1} b_{1}}{a_{2} b_{2}\left(1-c_{1}\right)} .
$$

Theorem 3.1. If $c_{1}>1$, then the line segment $\mathfrak{A}$ of equilibrium points is the global attractor for system (3.1). Further, any trajectory converges to an equilibrium point $\left(x_{*}, y_{*}, p_{*}\right) \in \mathfrak{A}$.

We note that system (3.1) is written in an implicit form. If $c_{1}>1$ as in Theorem 3.1, it is easy to rewrite this system as an explicit PWL map $\left(x_{t}, y_{t}, p_{t}\right)=f\left(x_{t-1}, y_{t-1}, p_{t-1}\right)$.

\subsection{Sticky Central Bank response}

The Central Bank policy can potentially exhibit stickiness too. To explore this scenario in this section we replace the Taylor rule (2.2) with the relation

$$
r_{t}=\mathfrak{p}_{\sigma}\left[c_{1} x+c_{2} y\right]_{t}+\xi_{t}
$$

also involving a play operator with an initial condition $r_{0}$ such that $\left|r_{0}-\left(c_{1} x_{0}+c_{2} y_{0}\right)\right| \leq \sigma$. The play operator $\mathfrak{p}_{\sigma}$ with a threshold $\sigma \geq 0$ (independent of $\rho$ ) in (3.3) should express the fact that the Central Bank's decisions do not immediately follow the instantaneous value of $c_{1} x_{t}+c_{2} y_{t}$, but they are activated only if the difference between $r_{t}$ and $c_{1} x_{t}+c_{2} y_{t}$ risks exceeding a given value $\sigma$. For $\sigma=0, \mathfrak{p}_{\sigma}$ is the identity mapping and (3.3) becomes (2.2).

It is worth noting that for $\sigma>0$ and a sequence $\left\{v_{t}\right\}, r_{t}=\mathfrak{p}_{\sigma}[v]_{t}$ is the sequence with minimal variation in the $\sigma$-neighborhood of $\left\{v_{t}\right\}$, that is, the implication

$$
\hat{r}_{0}=r_{0},\left|\hat{r}_{t}-v_{t}\right| \leq \sigma \quad \forall t \in \mathbb{N} \cup\{0\} \quad \Rightarrow \quad \sum_{t=1}^{T}\left|\hat{r}_{t}-\hat{r}_{t-1}\right| \geq \sum_{t=1}^{T}\left|r_{t}-r_{t-1}\right|
$$

holds for every sequence $\left\{\hat{r}_{t}\right\}$ and any $T \in \mathbb{N}$ as a special case of ([33], Thm. 1.2 and Cor. 1.6). The rule (3.3) can thus be interpreted as the optimal strategy requiring $r_{t}$ to stay in a $\sigma$-neighborhood of the input sequence while minimizing any "costs" incurred by varying the interest rate (and so this second application of the play operator can be considered a 'rational' variant of the underlying model in that the Central Bank is still engaging in a form of optimizing behavior).

Re-writing equation (3.3) equivalently as

$$
r_{t}=c_{1} x_{t}+c_{2} y_{t}+\Phi_{\sigma}\left(r_{t-1}-c_{1} x_{t-1}-c_{2} y_{t-1}\right)+\xi_{t},
$$

we obtain a 4-dimensional PWL system (2.1), (2.3), (3.5). In the absence of noise, i.e. when $\eta_{t}=\epsilon_{t}=\xi_{t}=0$ for all $t$, this system has the following set of equilibrium points:

$$
\begin{gathered}
\mathfrak{M}=\left\{\left(x_{*}, y_{*}, p_{*}, r_{*}\right): x_{*}=\kappa u+\frac{v}{c_{1}-1}, y_{*}=\frac{b_{1}}{b_{2}} u, p_{*}=(\kappa-1) u+\frac{v}{c_{1}-1},\right. \\
\left.r_{*}=\left(c_{1} \kappa+\frac{b_{1} c_{2}}{b_{2}}\right) u+\frac{v}{c_{1}-1}, \quad|u| \leq \rho,|v| \leq \sigma\right\} .
\end{gathered}
$$

The subset

$$
\mathfrak{A}_{0}=\left\{\left(x_{*}, y_{*}, p_{*}, r_{*}\right) \in \mathfrak{M}: v=0\right\}
$$


of $\mathfrak{M}$ can be considered as a natural embedding of the equilibrium set (3.2) into the 4-dimensional phase space of system (2.1), (2.3), (3.5).

It should be pointed out right away that stickiness in the Taylor rule can have a destabilizing effect on the equilibrium states. To see this, consider for simplicity the case when $\rho=0$, which implies $p=x$ and $s=0$, i.e. we remove stickiness in the inflation expectation. Note that the system is locally linear in a vicinity of every equilibrium point belonging to $\mathfrak{M}$ and satisfying $|v|<\sigma$. Further, a simple calculation shows that the determinant of the linearization at such equilibrium points equals $\left(1-a_{1}\right)\left(1-b_{1}\right) /\left(1-b_{1}-a_{2} b_{2}\right)$. If $1-b_{1}>$ $a_{2} b_{2}>a_{1}\left(1-b_{1}\right)$, then this determinant is greater than 1 , hence these equilibria are unstable (in particular, they are unstable in the important case of $a_{1}=0,1-b_{1}>a_{2} b_{2}$ ). Numerical examples of several attractors including periodic orbits of different periods, a quasiperiodic orbit or a union of two equilibrium points corresponding to $v= \pm \sigma$ (end points of the line segment of equilibrium points) can be found in [34].

We now estimate how far trajectories can deviate from the equlibrium set $\mathfrak{A}_{0}$ due to stickiness in the Taylor rule and a uniformly bounded noise.

Theorem 3.2. Consider the system (2.1), (2.3), (3.5) with uniformly bounded exogenous terms $\eta_{t}, \epsilon_{t}, \xi_{t}$ and with $c_{1}>1$. There exist constants $L_{1}, L_{2}$, which depend on the parameters $a_{1}, a_{2}, b_{1}, b_{2}, c_{1}, c_{2}$ but are independent of the threshold $\rho$ of the inflation expectation (see (2.3)), such that every trajectory satisfies

$$
\limsup _{t \rightarrow \infty}\left|x_{t}-x_{*}\left(s_{t}\right)\right|, \limsup _{t \rightarrow \infty}\left|y_{t}-y_{*}\left(s_{t}\right)\right| \leq L_{1} \sigma+L_{2} m,
$$

where $\left(x_{*}, y_{*}, p_{*}\right)(\cdot)$ is defined in $(3.2), s_{t}=x_{t}-p_{t}, \sigma$ is the threshold of the play operator in the central bank's policy (see (3.3)) and

$$
m=\sup _{t} \max \left\{\left|\epsilon_{t}\right|,\left|\eta_{t}\right|,\left|\xi_{t}\right|\right\}<\infty
$$

The proof presented below suggests an explicit upper bound for the coefficients $L_{1}, L_{2}$ in (3.7). Due to relations $p=x-s$ and (3.5), estimates (3.7) imply similar estimates for $p_{t}$ and $r_{t}$ :

$$
\begin{gathered}
\limsup _{t \rightarrow \infty}\left|p_{t}-p_{*}\left(s_{t}\right)\right| \leq L_{1} \sigma+L_{2} m, \\
\limsup _{t \rightarrow \infty}\left|r_{t}-c_{1} x_{*}\left(s_{t}\right)-c_{2} y_{*}\left(s_{t}\right)\right| \leq\left(c_{1}+c_{2}\right)\left(L_{1} \sigma+L_{2} m\right)+\sigma+m .
\end{gathered}
$$

According to Theorem 3.2, estimates (3.7)-(3.10) are uniform with respect to $\rho$. In particular, if $\sigma=0$ and hence system $(2.1),(2.3),(3.5)$ becomes equivalent to system $(2.1)-(2.3)$, then every trajectory converges to a neigborhood

$$
\mathfrak{A}(R)=\left\{(x, y, p): \min _{\left(x_{*}, y_{*}, p_{*}\right) \in \mathfrak{A}} \max \left\{\left|x-x_{*}\right|,\left|y-y_{*}\right|,\left|p-p_{*}\right|\right\} \leq R\right\}
$$

of the set (3.2) of equilibrium points, and the size of this neighborhood is proportional to the supremum norm of the noise terms, $R=L_{2} m$, and is independent of the threshold $\rho$ of inflation expectations (cf. Thm 3.1).

\subsection{A multi-agent model}

Model (2.1)-(2.3) can be easily extended to account for differing types of agent with different inflation rate expectation thresholds. To this end, we replace the simple relationship (2.5) between $p_{t}$ and $x_{t}$ (which is 
equivalent to (2.3)) with the equation

$$
p_{t}=\sum_{i=1}^{n} \nu_{i} \mathfrak{p}_{\rho_{i}}\left[\beta_{i}, x\right]_{t}
$$

Here the play operator $\mathfrak{p}_{\rho_{i}}$ models the expectation of inflation by the $i$-th agent; $p_{t}$ is the aggregate expectation of inflation; $\nu_{i}>0$ is a weight measuring the contribution of that agent's expectation of inflation to the aggregate quantity; $\rho_{i}$ is an individual threshold characterizing the behavior of the $i$-th agent; $\beta_{i}$ is the initial condition for the corresponding play operator; and we assume the ordering $0<\rho_{1}<\cdots<\rho_{n}$.

Operator (3.11) is known as a (discrete) Prandtl-Ishlinskii (PI) operator [26, 30, 46]. ${ }^{2}$ In analysing this operator, it is convenient to restrict the set of initial conditions of the play operators in (3.11). In what follows, we assume that $\left|\beta_{1}\right|<\rho_{1}$ and $\left|\beta_{i}-\beta_{i-1}\right| \leq \rho_{i}-\rho_{i-1}$ for all $2 \leq i \leq n$. Further, the coefficients $\nu_{i}$ are assumed to fulfill the condition

$$
\nu_{i}>0 \text { for } i=1, \ldots, n, \quad \nu_{0}:=1-\sum_{i=1}^{n} \nu_{i} \geq 0 .
$$

It will be useful to define the quantity $s_{t}$ in a similar way to $(2.7)$, that is

$$
s_{t}=x_{t}-p_{t}=\nu_{0} x_{t}+\sum_{i=1}^{n} \nu_{i} \mathfrak{s}_{i}\left[\beta_{i}, x\right]_{t},
$$

where $\mathfrak{s}_{\rho_{i}}\left[\beta_{i}, x\right]_{t}=x_{t}-\mathfrak{p}_{\rho_{i}}\left[\beta_{i}, x\right]_{t}$ for all $t \in \mathbb{N} \cup\{0\}$.

Equations (2.1), (2.2), (3.11) form an $(n+2)$-dimensional PWL system. Similarly, if the Taylor rule $(2.2)$ is replaced with its sticky counterpart (3.5), we obtain a PWL system of dimension $n+3$. One can formulate natural analogs of Theorems 3.1, 3.2 for these systems. For example, let us consider the analog of Theorem 3.1 for the autonomous system

$$
\begin{aligned}
& x_{t}=b_{1} p_{t}+\left(1-b_{1}\right) x_{t-1}+b_{2} y_{t}, \\
& y_{t}=\left(1-a_{1}\right) y_{t-1}-a_{2}\left(c_{1} x_{t}+c_{2} y_{t}-p_{t}\right)
\end{aligned}
$$

coupled with formula (3.11) for the aggregate expectation of inflation.

Theorem 3.3. If $c_{1}>1$, then any trajectory of system (3.11), (3.14) converges to an equilibrium point of this system.

We note that equilibrium points of system (3.11), (3.14) form an $n$-dimensional parallelepiped in its phase space. The proof of Theorem 3.3 uses the constructions from the proof of Theorem 3.1 but additionally relies on an inversion formula for the Prandtl-Ishlinskii operator. This proof also shows a possible way to extend Theorem 3.2 to the multi-agent model (2.1), (3.5), (3.11) with sticky inflation expectation and exogenous noise.

\section{Proofs}

\subsection{Play and stop operators}

For the reader's convenience, we summarize here some well-known properties of the discrete time stop operator $s_{t}=\mathfrak{s}_{\rho}\left[s_{0}, x\right]_{t}$ and play operator $p_{t}=\mathfrak{p}_{\rho}\left[s_{0}, x\right]_{t}$ which are needed in the sequel.

\footnotetext{
${ }^{2}$ The continuous-time counterpart of this operator is widely used as a friction model in mechanical applications [39] as well as for modeling elastoplastic systems [35], constitutive laws of smart materials [2], and material fatigue [50].
} 
Lemma 4.1. Let $\left\{x_{t} ; t \in \mathbb{N} \cup\{0\}\right\}$ be a given sequence. Then $p_{t}, s_{t}$ satisfy (2.5), (2.7) if and only if $\left|s_{t}\right| \leq \rho$ for all $t \in \mathbb{N} \cup\{0\}$ and the variational inequality

$$
\left(p_{t}-p_{t-1}, s_{t}-z\right) \geq 0 \quad \forall t \in \mathbb{N} \cup\{0\}
$$

holds for every $z \in[-\rho, \rho]$.

Proof. Relations (2.5), (2.7) are equivalent to the series of implications

$$
p_{t}-p_{t-1}>0 \Rightarrow p_{t}=x_{t}-\rho \Rightarrow s_{t}=\rho, p_{t}-p_{t-1}<0 \Rightarrow p_{t}=x_{t}+\rho \Rightarrow s_{t}=-\rho,
$$

which is in turn equivalent to (4.1) under the condition $\left|s_{t}\right| \leq \rho$ for all $t \in \mathbb{N}$.

For a generic sequence $\left\{z_{t} ; t \in \mathbb{N} \cup\{0\}\right\}$, we introduce the notation

$$
\nabla_{t} z:=z_{t}-z_{t-1} \quad \text { if } \quad t \geq 1 ; \quad \nabla_{t}^{2} z=z_{t}-2 z_{t-1}+z_{t-2} \quad \text { if } t \geq 2 .
$$

We will systematically use the identity

$$
\nabla_{t} z z_{t}=\frac{1}{2}\left(z_{t}^{2}-z_{t-1}^{2}\right)+\frac{1}{2}\left(\nabla_{t} z\right)^{2}
$$

Choosing in (4.1) the value $z=s_{t-1}$, we obtain $\nabla_{t} p \nabla_{t} s \geq 0$, hence

$$
\nabla_{t} x \nabla_{t} s \geq\left(\nabla_{t} s\right)^{2} .
$$

Furthermore, summing the inequalities

$$
\begin{aligned}
\left(p_{t}-p_{t-1}\right)\left(s_{t}-s_{t-1}\right) & \geq 0, \\
\left(p_{t-1}-p_{t-2}\right)\left(s_{t-1}-s_{t}\right) & \geq 0,
\end{aligned}
$$

which follow from (4.1) with the choices $z=s_{t-1}$ and $z=s_{t}$, respectively, we obtain $\nabla_{t}^{2} p \nabla_{t} s \geq 0$, hence

$$
\nabla_{t}^{2} x \nabla_{t} s \geq \nabla_{t}^{2} s \nabla_{t} s=\frac{1}{2}\left(\left(\nabla_{t} s\right)^{2}-\left(\nabla_{t-1} s\right)^{2}\right)+\frac{1}{2}\left(\nabla_{t}^{2} s\right)^{2}
$$

and similarly

$$
\nabla_{t}^{2} x \nabla_{t} x=\frac{1}{2}\left(\left(\nabla_{t} x\right)^{2}-\left(\nabla_{t-1} x\right)^{2}\right)+\frac{1}{2}\left(\nabla_{t}^{2} x\right)^{2},
$$

which is a special case of identity (4.3) with $z_{t}=\nabla_{t} s$ and $z_{t}=\nabla_{t} x$.

Lemma 4.2. For a given sequence $\left\{x_{t} ; t \in \mathbb{N} \cup\{0\}\right\}$, let $p_{t}=\mathfrak{p}_{\rho}\left[s_{0}, x\right]_{t}, s_{t}=x_{t}-p_{t}$ with some given initial condition $s_{0} \in[-\rho, \rho]$. Let $q_{t}=x_{t}+\delta s_{t}=(1+\delta) x_{t}-\delta p_{t}$ for some $\delta>-1$. Then

$$
x_{t}=\frac{1}{1+\delta}\left(q_{t}+\delta \mathfrak{p}_{(1+\delta) \rho}\left[(1+\delta) s_{0}, q\right]_{t}\right)
$$

Proof. We have $q_{t}-p_{t}=(1+\delta) s_{t}$, hence $\left|q_{t}-p_{t}\right| \leq(1+\delta) \rho$, and

$$
\left(p_{t}-p_{t-1}, q_{t}-p_{t}-(1+\delta) \rho x\right) \geq 0 \quad \forall t \in \mathbb{N} \forall|x| \leq 1 .
$$


By Lemma 4.1, this implies that $p_{t}=\mathfrak{p}_{(1+\delta) \rho}\left[(1+\delta) s_{0}, q\right]_{t}$ and the assertion follows.

Lemma 4.3. For a given sequence $\left\{x_{t} ; t \in \mathbb{N} \cup\{0\}\right\}$, put $p_{t}=\mathfrak{p}_{\rho}\left[s_{0}, x\right]_{t}$ with some given initial condition $s_{0} \in[-\rho, \rho]$. Then for every $t, j \in \mathbb{N}$ we have

$$
\left|p_{t+j}-p_{t}\right| \leq \max _{i=1, \ldots, j}\left\{\left|x_{t+i}-x_{t}\right|\right\}
$$

Proof. We fix $t \in \mathbb{N} \cup\{0\}, J \in \mathbb{N}$ and for $j=0,1, \ldots, J$ set

$$
S_{j}=\max \left\{\left|p_{t+j}-p_{t}\right|^{2}, \max _{i=1, \ldots, J}\left\{\left|x_{t+i}-x_{t}\right|^{2}\right\}\right\}
$$

The proof will be complete if we prove that the sequence $\left\{S_{j}\right\}$ is nonincreasing for $j=0,1, \ldots, J$. Indeed, then $S_{J} \leq S_{0}$, which is precisely the desired statement.

Assume for contradiction that $S_{j}>S_{j-1}$ for some $j=1, \ldots, J$. Then

$$
\begin{aligned}
\left|p_{t+j}-p_{t}\right| & >\max _{i=1, \ldots, J}\left\{\left|x_{t+i}-x_{t}\right|\right\}, \\
\left(p_{t+j}-p_{t}\right)^{2} & >\left(p_{t+j-1}-p_{t}\right)^{2} .
\end{aligned}
$$

Inequality (4.11) can be equivalently written as

$$
\left(p_{t+j}-p_{t+j-1}\right)\left(p_{t+j}-p_{t}\right)>\frac{1}{2}\left(p_{t+j}-p_{t+j-1}\right)^{2}>0 .
$$

We now replace in (4.1), using $t+j$ instead of $t$, the element $z$ by $s_{t}$ and obtain

$$
\left(p_{t+j}-p_{t+j-1}\right)\left(s_{t+j}-s_{t}\right) \geq 0,
$$

hence, combining (4.12) with (4.13), we have

$$
\left(p_{t+j}-p_{t}\right)\left(s_{t+j}-s_{t}\right) \geq 0,
$$

which implies that

$$
\left(p_{t+j}-p_{t}\right)^{2} \leq\left(p_{t+j}-p_{t}\right)\left(x_{t+j}-x_{t}\right)
$$

contradicting (4.10). This completes the proof of Lemma 4.3.

\subsection{Long time asymptotics}

This section is devoted to the study of the asymptotic behavior of system (2.1), (3.3) as $t \rightarrow \infty$. Put $z_{t}=$ $\mathfrak{s}_{\sigma}\left[s_{0}, c_{1} p+c_{2} y\right]_{t}=c_{1} x_{t}+c_{2} y_{t}-r_{t}+\xi_{t}$. This allows us, with the notation (4.2), to rewrite (2.1), (3.3) in the form

$$
\begin{aligned}
\left(1-a_{1}\right) \nabla_{t} y+\left(a_{1}+a_{2} c_{2}\right) y_{t}+a_{2}\left(c_{1}-1\right) x_{t}+a_{2} s_{t} & =a_{2}\left(z_{t}-\xi_{t}\right)+\epsilon_{t} \\
\left(1-b_{1}\right) \nabla_{t} x+b_{1} s_{t}-b_{2} y_{t} & =\eta_{t}
\end{aligned}
$$

As a consequence of (4.14), we have

$$
\left(1-b_{1}\right) \nabla_{t}^{2} x+b_{1} \nabla_{t} s-b_{2} \nabla_{t} y=\nabla_{t} \eta
$$


with the notation (4.2). This enables us to eliminate $y_{t}$ from the system (4.14) and reformulate it as a second order equation

$$
\nabla_{t}^{2} x+A \nabla_{t} x+B \nabla_{t} s+C x_{t}+D s_{t}=h_{t}, \quad t \geq 2
$$

with positive constants

$$
A=\frac{a_{1}+a_{2} c_{2}}{1-a_{1}}, B=\frac{b_{1}}{1-b_{1}}, C=\frac{a_{2} b_{2}\left(c_{1}-1\right)}{\left(1-a_{1}\right)\left(1-b_{1}\right)}, D=\frac{b_{1}\left(a_{1}+a_{2} c_{2}\right)+a_{2} b_{2}}{\left(1-a_{1}\right)\left(1-b_{1}\right)},
$$

and with the right-hand side

$$
h_{t}=\frac{1}{\left(1-a_{1}\right)\left(1-b_{1}\right)}\left(\left(1-a_{1}\right) \nabla_{t} \eta+b_{2}\left(a_{2}\left(z_{t}-\xi_{t}\right)+\epsilon_{t}\right)+\left(a_{1}+a_{2} c_{2}\right) \eta_{t}\right) .
$$

The sequence $\left\{h_{t}\right\}$ contains the term $z_{t}$ which is bounded above by $\sigma$, and the noise terms $\epsilon_{t}, \xi_{t}, \eta_{t}$, and $\nabla_{t} \eta$.

Equation (4.15) always has a solution $x_{t}$ at each time step $t$, since the right-hand side of (4.15) is for each fixed $t$ a bounded function of $x_{t}$ and the left hand side is an increasing piecewise linear function of $x_{t}$. In some cases, the solution may not be unique if the coefficient $D^{*}:=a_{2} b_{2} /\left(\left(1-a_{1}\right)\left(1-b_{1}\right)\right)$ in front of $x_{t}$ on the right-hand side is large. Our computations below show, however, that all solutions have the same asymptotic convergence towards a small neighborhood of a particular equilibrium point depending on the trajectory.

\subsubsection{Auxiliary estimate 1}

We put

$$
q_{t}:=C x_{t}+D s_{t},
$$

and multiply the equation (4.15) by $\nabla_{t} q=C \nabla_{t} x+D \nabla_{t} s$. Putting

$$
V_{t}^{1}:=\frac{1}{2}\left(C\left(\nabla_{t} x\right)^{2}+D\left(\nabla_{t} s\right)^{2}+q_{t}^{2}\right)
$$

and using the relations (4.3)-(4.4) we obtain that

$$
V_{t}^{1}-V_{t-1}^{1}+\frac{C}{2}\left(\nabla_{t}^{2} x\right)^{2}+\frac{D}{2}\left(\nabla_{t}^{2} s\right)^{2}+A C\left(\nabla_{t} x\right)^{2}+(B C+A D+B D)\left(\nabla_{t} s\right)^{2}+\frac{1}{2}\left(\nabla_{t} q\right)^{2} \leq h_{t} \nabla_{t} q .
$$

\subsubsection{Auxiliary estimate 2}

We now rewrite (4.15) in the form

$$
\nabla_{t}^{2} x+\frac{A}{C} \nabla_{t} q+\left(B-\frac{A D}{C}\right) \nabla_{t} s+q_{t}=h_{t}
$$

with $q_{t}$ given by (4.18), and multiply it by $q_{t}$. We use (4.3) again and find constants $E, F>0$ depending on $A, B, C, D$ such that

$$
\begin{aligned}
\nabla_{t} x q_{t}-\nabla_{t-1} x q_{t-1}+\frac{A}{2 C}\left(q_{t}^{2}-q_{t-1}^{2}\right)+\frac{1}{2} q_{t}^{2} & \leq\left(\nabla_{t} x-\nabla_{t}^{2} x\right) \nabla_{t} q+E\left(\nabla_{t} s\right)^{2}-\frac{A}{2 C}\left(\nabla_{t} q\right)^{2}+h_{t} q_{t} \\
& \leq F\left(\frac{1}{2}\left(\nabla_{t}^{2} x\right)^{2}+A\left(\nabla_{t} x\right)^{2}\right)+h_{t} q_{t} .
\end{aligned}
$$


We now set

$$
V_{t}^{0}:=\nabla_{t} x q_{t}+\frac{A}{2 C} q_{t}^{2}
$$

so that (4.22) has the form

$$
V_{t}^{0}-V_{t-1}^{0}+\frac{1}{2} q_{t}^{2} \leq F\left(\frac{1}{2}\left(\nabla_{t}^{2} x\right)^{2}+A\left(\nabla_{t} x\right)^{2}\right)+h_{t} q_{t}
$$

Finally, we choose $\lambda>0$ such that $\lambda F<C$, and $\lambda V_{t}^{0} \geq-\frac{1}{2} V_{t}^{1}$ for all $t \in \mathbb{N}$, and put

$$
W_{t}=V_{t}^{1}+\lambda V_{t}^{0}
$$

Then, from (4.20) and (4.24), there exists a constant $\mu>0$ such that

$$
W_{t}-W_{t-1}+\mu W_{t} \leq L\left|h_{t}\right| \sqrt{W_{t}}
$$

for all $t \in \mathbb{N}$ as a consequence of (4.19), (4.20), and (4.24), with some constant $L>0$.

\subsubsection{Asymptotic behavior: Proof of Theorems 3.1-3.2}

Let $c_{1}>1$. If $h_{t}=0$, that is, no noise is present and the reaction of the Central Bank is instantaneous with $\sigma=0$, then $W_{t}$ is a Lyapunov function of the system which decays exponentially to 0 . In particular, $q_{t}$ defined by (4.18), converges exponentially to $q_{\infty}=0$. Then, it follows from Lemmas 4.2 and 4.3 that $x_{t}$ converges to some value $x_{\infty}$, hence $s_{t}=\mathfrak{s}_{\rho}\left[s_{0}, x\right]_{t}$ converges to some $s_{\infty}$ such that

$$
C x_{\infty}+D s_{\infty}=0
$$

and $p_{t}=x_{t}-s_{t} \rightarrow x_{\infty}-s_{\infty}$. Further, equations (3.1) imply that $y_{t} \rightarrow y_{\infty}$ and the point $\left(x_{\infty}, y_{\infty}, p_{\infty}\right)$ belongs to the set (3.2). This completes the proof of Theorem 3.1.

In the case of a general right-hand side $h_{t}$, we have for every $t>T>0$, as a consequence of (4.26), that

$$
W_{t} \leq(1+\mu)^{-T} W_{t-T}+L \sum_{j=t-T+1}^{t}\left|h_{j}\right| \sqrt{W_{j}}(1+\mu)^{j-1-t}
$$

Assume that there exists $T>0$ such that for all $t_{0} \in \mathbb{N}$ we have

$$
\frac{1}{T} \sum_{j=t_{0}+1}^{t_{0}+T}\left|h_{j}\right| \leq \hat{\sigma}
$$

Then it follows from (4.28) that

$$
W_{t} \leq(1+\mu)^{-T} W_{t-T}+\frac{L T \hat{\sigma}}{1+\mu} \max _{j=t-T+1, \ldots, t} \sqrt{W_{j}}
$$


for all $t>T$. Assume first that there exists $t>T$ such that $W_{t} \geq W_{j}$ for all $j=t-T, \ldots, t$. Then (4.30) yields that

$$
W_{t} \leq(1+\mu)^{-T} W_{t}+\frac{L T \hat{\sigma}}{1+\mu} \sqrt{W_{t}},
$$

giving

$$
W_{t} \leq\left(\frac{L T \hat{\sigma}(1+\mu)^{T-1}}{(1+\mu)^{T}-1}\right)^{2}
$$

This implies in particular that $W_{t}$ is bounded and we put

$$
W^{*}:=\limsup _{t \rightarrow \infty} W_{t}<\infty
$$

For an arbitrary $\delta>0$ we find $t_{0}$ sufficiently large such that for all $t>t_{0}-T$ we have $W_{t} \leq W^{*}+\delta$. Then for $t>t_{0}$ we obtain from (4.30) that

$$
W_{t} \leq(1+\mu)^{-T}\left(W^{*}+\delta\right)+\frac{L T \hat{\sigma}}{1+\mu} \sqrt{W^{*}+\delta},
$$

hence,

$$
W^{*} \leq(1+\mu)^{-T}\left(W^{*}+\delta\right)+\frac{L T \hat{\sigma}}{1+\mu} \sqrt{W^{*}+\delta},
$$

that is,

$$
W^{*}+\delta \leq \frac{L T \hat{\sigma}(1+\mu)^{T-1}}{(1+\mu)^{T}-1} \sqrt{W^{*}+\delta}+\frac{\delta(1+\mu)^{T}}{(1+\mu)^{T}-1}
$$

and we conclude that

$$
W^{*} \leq\left(\frac{L T \hat{\sigma}(1+\mu)^{T-1}}{(1+\mu)^{T}-1}\right)^{2}+\delta \frac{(1+\mu)^{T}+1}{(1+\mu)^{T}-1} .
$$

Since $\delta>0$ is arbitrary, we obtain that

$$
\limsup _{t \rightarrow \infty} W_{t} \leq\left(\frac{L T \hat{\sigma}(1+\mu)^{T-1}}{(1+\mu)^{T}-1}\right)^{2} .
$$

Estimate (4.32) gives a uniform upper bound for the value of $\lim _{\sup _{t \rightarrow \infty}} W_{t}$ independent of the initial condition. In particular, if $\left|h_{t}\right| \leq \hat{\sigma}$ for all $t \in \mathbb{N}$, then formula (4.32) holds with $T=1$, that is,

$$
\limsup _{t \rightarrow \infty} W_{t} \leq\left(\frac{L \hat{\sigma}}{\mu}\right)^{2}
$$


Since by construction $\left(C x_{t}+D s_{t}\right)^{2}=q_{t}^{2} \leq 2 V_{t}^{1} \leq 4 W_{t}^{1}$ and by definition $x_{*}\left(s_{t}\right)=\kappa s_{t}=-s_{t} D / C$, it follows that

$$
\limsup _{t \rightarrow \infty}\left(x_{t}-x_{*}\left(s_{t}\right)\right)^{2} \leq\left(\frac{2 L \hat{\sigma}}{\mu C}\right)^{2} .
$$

Condition (3.8) of Theorem 3.2 and the definition of $h_{t}$ (see (4.17)) imply that the estimate $\left|h_{t}\right| \leq \hat{\sigma}$ holds with $\hat{\sigma}=L^{\prime} \sigma+L^{\prime \prime} m$, hence (4.34) implies the first of the estimates (3.7). Further, the first equation in (2.1) implies that

$$
y_{t}-y_{*}\left(s_{t}\right)=y_{t}-\frac{b_{1} s_{t}}{b_{2}}=\frac{1-b_{1}}{b_{2}} \nabla_{t} x-\frac{\eta_{t}}{b_{2}},
$$

hence the second of the estimates (3.7) follows from the relations $C\left(\nabla_{t} x\right)^{2} \leq 2 V_{t}^{1} \leq 4 W_{t}$ and (4.33) combined with $\hat{\sigma}=L^{\prime} \sigma+L^{\prime \prime} m$ and (3.8). This completes the proof of Theorem 3.2.

A few remarks are in order. First, estimate (4.32) can be useful if system (2.1) is obtained as a time discretization of a continuous time system and the increments of noise are of class $\ell_{l o c}^{1}$. Second, counterparts of Theorems 3.1-3.2 can be obtained by the same method if $p_{t}$ is allowed to depend also on $y_{t}$, for example, if $p_{t}=\mathfrak{p}_{\rho}\left[s_{0}, x+\delta y\right]_{t}$ with a small $\delta>0$.

\subsection{Stability of the multi-agent model}

As mentioned earlier, the proof of Theorem 3.3 is parallel to the proof of Theorem 3.1 but additionally relies on an inversion formula for the Prandtl-Ishlinskii operator. We start by deriving the inverse operator.

\subsubsection{Inversion of the discrete-time Prandtl-Ishlinskii operator}

The main tool in our analysis is the following identity which, being inspired by the developments in ([30], $\S 34)$, is essentially due to M. Brokate, see ([9], Prop. 2.2.16).

Lemma 4.4 (Brokate identity). Let $\rho>0, \sigma>0, \beta \in[-\rho, \rho], \gamma \in[-\sigma, \sigma]$, and $x \in \mathcal{S}$ be given. Put $p=\mathfrak{p}_{\rho}[\beta, x]$, $q=\mathfrak{p}_{\sigma}[\gamma, p]$. Then $q=\mathfrak{p}_{\rho+\sigma}[\beta+\gamma, x]$. Moreover, the implication

$$
q_{t}-q_{t-1} \neq 0 \Longrightarrow\left(p_{t}-p_{t-1}\right)\left(q_{t}-q_{t-1}\right)>0
$$

holds for every $t \in \mathbb{N}$.

Proof. Put $\varphi=\mathfrak{p}_{\rho+\sigma}[\beta+\gamma, x]$. We have by definition

$$
\begin{aligned}
\left(p_{t}-p_{t-1}\right)\left(x_{t}-p_{t}-\rho z\right) & \geq 0 \\
\left(q_{t}-q_{t-1}\right)\left(p_{t}-q_{t}-\sigma z\right) & \geq 0 \\
\left(\varphi_{t}-\varphi_{t-1}\right)\left(x_{t}-\varphi_{t}-(\rho+\sigma) z\right) & \geq 0
\end{aligned}
$$

for all $t \in \mathbb{N}$ and all $z \in[-1,1]$. In (4.37), we may choose $\sigma z=p_{t-1}-q_{t-1}$ and obtain

$$
\left(q_{t}-q_{t-1}\right)^{2} \leq\left(q_{t}-q_{t-1}\right)\left(p_{t}-p_{t-1}\right),
$$

and the implication (4.35) follows. In particular, the inequality (4.36) remains valid if we replace $p_{t}-p_{t-1}$ with $q_{t}-q_{t-1}$, that is,

$$
\left(q_{t}-q_{t-1}\right)\left(x_{t}-p_{t}-\rho z\right) \geq 0
$$


for all $t \in \mathbb{N}$ and all $z \in[-1,1]$. Adding (4.39) to (4.37) yields

$$
\left(q_{t}-q_{t-1}\right)\left(x_{t}-q_{t}-(\rho+\sigma) z\right) \geq 0
$$

for all $t \in \mathbb{N}$ and all $z \in[-1,1]$. We have indeed $\left|x_{t}-q_{t}\right| \leq\left|x_{t}-p_{t}\right|+\left|p_{t}-q_{t}\right| \leq \rho+\sigma$, hence we may replace $(\rho+\sigma) z$ in (4.38) with $x_{t}-q_{t}$, in (4.40) with $x_{t}-\varphi_{t}$, and sum the two inequalities to obtain

$$
\left(\left(q_{t}-\varphi_{t}\right)-\left(q_{t-1}-\varphi_{t-1}\right)\right)\left(q_{t}-\varphi_{t}\right) \leq 0
$$

From (4.41) and (4.3) for $z=q-\varphi$ it thus follows $\left(q_{t}-\varphi_{t}\right)^{2} \leq\left(q_{0}-\varphi_{0}\right)^{2}$ for all $t \in \mathbb{N}$. We have $\varphi_{0}=x_{0}-\beta-\gamma$, $q_{0}=p_{0}-\gamma=x_{0}-\beta-\gamma=\varphi_{0}$, and this completes the proof.

We now recall the definition of a Prandtl-Ishlinskii operator as a linear combination of play operators. More specifically, let $n \in \mathbb{N}, \nu_{0}, \ldots, \nu_{n} \in \mathbb{R}, 0=\rho_{0}<\rho_{1}<\cdots<\rho_{n}$, and $\beta_{i} \in\left[-\rho_{i}, \rho_{i}\right]$ be given numbers (in particular, $\left.\beta_{0}=0\right)$. For $x \in \mathcal{S}$ and $\beta=\left(\beta_{1}, \ldots, \beta_{n}\right)$ we put

$$
\mathcal{F}[\beta, x]=\sum_{i=0}^{n} \nu_{i} \mathfrak{p}_{\rho_{i}}\left[\beta_{i}, x\right]
$$

with the convention $\mathfrak{p}_{0}[0, x]=x$. The mapping $\mathcal{F}:\left[-\rho_{1}, \rho_{1}\right] \times \cdots \times\left[-\rho_{n}, \rho_{n}\right] \times \mathcal{S} \rightarrow \mathcal{S}$ is called a (time and memory discrete) Prandtl-Ishlinskii operator.

Hypothesis 4.5. Let $\mathcal{F}$ be a Prandtl-Ishlinskii operator as in (4.42). The following conditions are assumed to hold:

(i) $\left|\beta_{i}-\beta_{i-1}\right| \leq \rho_{i}-\rho_{i-1}$ for $i=1, \ldots, n$;

(ii) $A_{i}:=\sum_{j=0}^{i} \nu_{j}>0$ for $i=0, \ldots, n$.

We prove the following statement which shows that the inverse of a discrete Prandtl-Ishlinskii operator is again a Prandtl-Ishlinskii operator. In the continuous case, this result goes back to [36]. Finite collections of stops have been considered in [47]. A substantially more general situation in the space of regulated functions is considered in $[37,38]$. In fact, the explicit inversion formula presented below can also be deduced from ([37], Cor. 3.3) which uses deeper results from the Kurzweil integration theory. In the discrete case, there exists an elementary proof that we present here. Note that Lemma 4.2 is a special case of Theorem 4.6 in the case $n=1$.

Theorem 4.6. Let Hypothesis 4.5 hold. Define $B_{i}:=1 / A_{i}$ and

$$
\begin{gathered}
\sigma_{0}=\gamma_{0}=0, \quad \sigma_{i}-\sigma_{i-1}=A_{i-1}\left(\rho_{i}-\rho_{i-1}\right), \quad \gamma_{i}-\gamma_{i-1}=A_{i-1}\left(\beta_{i}-\beta_{i-1}\right), \\
\zeta_{0}:=B_{0}, \quad \zeta_{i}:=B_{i}-B_{i-1}
\end{gathered}
$$

for $i=1, \ldots, n$. For an arbitrary $x \in \mathcal{S}$ put

$$
v=\sum_{i=0}^{n} \nu_{i} \mathfrak{p}_{\rho_{i}}\left[\beta_{i}, x\right] .
$$

Then,

$$
x=\sum_{i=0}^{n} \zeta_{i} \mathfrak{p}_{\sigma_{i}}\left[\gamma_{i}, v\right] .
$$


We start with an auxiliary identity.

Proposition 4.7. Let the hypotheses of Theorem 4.6 hold. For $i=1, \ldots, n$ put

$$
x^{(i)}=\mathfrak{p}_{\rho_{i}-\rho_{i-1}}\left[\beta_{i}-\beta_{i-1}, x^{(i-1)}\right], \quad v^{(i)}=\mathfrak{p}_{\sigma_{i}-\sigma_{i-1}}\left[\gamma_{i}-\gamma_{i-1}, v^{(i-1)}\right]
$$

with $x^{(0)}=x, v^{(0)}=v$. Then for $j=1, \ldots, n$ we have

$$
v^{(j)}=A_{j} x^{(j)}+\sum_{i=j+1}^{n} \nu_{i} x^{(i)} .
$$

Proof of Proposition 4.7. The definition of the play states that

$$
\begin{gathered}
\left(x_{t}^{(i)}-x_{t-1}^{(i)}\right)\left(x_{t}^{(i-1)}-x_{t}^{(i)}-\left(\rho_{i}-\rho_{i-1}\right) z\right) \geq 0, \\
\left(v_{t}^{(i)}-v_{t-1}^{(i)}\right)\left(v_{t}^{(i-1)}-v_{t}^{(i)}-\left(\sigma_{i}-\sigma_{i-1}\right) z\right) \geq 0
\end{gathered}
$$

for all $i=1, \ldots n, t \in \mathbb{N}$, and $|z| \leq 1$. For $j=1, \ldots, n$ put

$$
\left(\varphi_{0}^{(j)}, \varphi_{1}^{(j)}, \ldots\right)=\varphi^{(j)}:=A_{j} x^{(j)}+\sum_{i=j+1}^{n} \nu_{i} x^{(i)} .
$$

Then we have

$$
\varphi^{(0)}=v^{(0)}, \quad \varphi_{t}^{(i)}-\varphi_{t}^{(i-1)}=A_{i-1}\left(x_{t}^{(i)}-x_{t}^{(i-1)}\right) .
$$

Hence, we may choose in (4.43)-(4.44) suitable values of $z$ in order to obtain

$$
\begin{aligned}
& \left(x_{t}^{(i)}-x_{t-1}^{(i)}\right)\left(\varphi_{t}^{(i-1)}-\varphi_{t}^{(i)}-v_{t}^{(i-1)}+v_{t}^{(i)}\right) \geq 0, \\
& \left(v_{t}^{(i)}-v_{t-1}^{(i)}\right)\left(v_{t}^{(i-1)}-v_{t}^{(i)}-\varphi_{t}^{(i-1)}+\varphi_{t}^{(i)}\right) \geq 0
\end{aligned}
$$

for $i=1, \ldots n$ and $t \in \mathbb{N}$. We now use the implication (4.35) to conclude that for all $n \geq j \geq i \geq 1$ we have

$$
\begin{aligned}
& \left(x_{t}^{(j)}-x_{t-1}^{(j)}\right)\left(\varphi_{t}^{(i-1)}-\varphi_{t}^{(i)}-v_{t}^{(i-1)}+v_{t}^{(i)}\right) \geq 0, \\
& \left(v_{t}^{(j)}-v_{t-1}^{(j)}\right)\left(v_{t}^{(i-1)}-v_{t}^{(i)}-\varphi_{t}^{(i-1)}+\varphi_{t}^{(i)}\right) \geq 0 .
\end{aligned}
$$

Summing the above inequalities over $i=1, \ldots, j$ yields (note that $\varphi^{(0)}=v^{(0)}$ by $(4.45)$ )

$$
\left(x_{t}^{(j)}-x_{t-1}^{(j)}\right)\left(v_{t}^{(j)}-\varphi_{t}^{(j)}\right) \geq 0, \quad\left(v_{t}^{(j)}-v_{t-1}^{(j)}\right)\left(\varphi_{t}^{(j)}-v_{t}^{(j)}\right) \geq 0
$$

for $j=1, \ldots, n, t \in \mathbb{N}$. In particular, for $j=n$, we have $\varphi^{(n)}=A_{n} x^{(n)}$. Multiplying the first inequality of (4.48) by $A_{n}$ and adding the second inequality yields

$$
\left(\varphi_{t}^{(n)}-v_{t}^{(n)}-\varphi_{t-1}^{(n)}+v_{t-1}^{(n)}\right)\left(\varphi_{t}^{(n)}-v_{t}^{(n)}\right) \leq 0
$$

for all $t \in \mathbb{N}$. Hence, by (4.3),

$$
\left(\varphi_{t}^{(n)}-v_{t}^{(n)}\right)^{2} \leq\left(\varphi_{0}^{(n)}-v_{0}^{(n)}\right)^{2} .
$$


Note that by $(4.45), \varphi_{0}^{(i)}-\varphi_{0}^{(i-1)}=A_{i-1}\left(x_{0}^{(i)}-x_{0}^{(i-1)}\right)=A_{i-1}\left(\beta_{i}-\beta_{i-1}\right)=\gamma_{i}-\gamma_{i-1}=v_{0}^{(i)}-v_{0}^{(i-1)}$, hence

$$
\varphi_{0}^{(i)}=v_{0}^{(i)} \quad \text { for } i=0, \ldots n
$$

and from (4.49) it follows that

$$
\varphi^{(n)}=v^{(n)}
$$

We now continue by backward induction and assume that $\varphi^{(j)}=v^{(j)}$ for some $2 \leq j \leq n$. From (4.48) written with $j-1$ instead of $j$ we obtain, using the induction hypothesis, that

$$
\begin{aligned}
& -\left(x_{t}^{(j-1)}-x_{t-1}^{(j-1)}\right)\left(v_{t}^{(j)}-\varphi_{t}^{(j)}-v_{t}^{(j-1)}+\varphi_{t}^{(j-1)}\right) \geq 0, \\
& -\left(v_{t}^{(j-1)}-v_{t-1}^{(j-1)}\right)\left(\varphi_{t}^{(j)}-v_{t}^{(j)}-\varphi_{t}^{(j-1)}+v_{t}^{(j-1)}\right) \geq 0 .
\end{aligned}
$$

We now add (4.53) to (4.47), multiply the sum of (4.52) with (4.46) by $A_{j-1}$, use (4.45), and sum the two resulting inequalities to conclude that

$$
\left(\left(v_{t}^{(j)}-\varphi_{t}^{(j)}-v_{t}^{(j-1)}+\varphi_{t}^{(j-1)}\right)-\left(v_{t-1}^{(j)}-\varphi_{t-1}^{(j)}-v_{t-1}^{(j-1)}+\varphi_{t-1}^{(j-1)}\right)\right)\left(v_{t}^{(j)}-\varphi_{t}^{(j)}-v_{t}^{(j-1)}+\varphi_{t}^{(j-1)}\right) \leq 0 .
$$

This is an inequality of type (4.3) which, together with the induction assumption $\varphi^{(j)}=v^{(j)}$ yields

$$
\left(v_{t}^{(j-1)}-\varphi_{t}^{(j-1)}\right)^{2} \leq\left(v_{t-1}^{(j-1)}-\varphi_{t-1}^{(j-1)}\right)^{2} .
$$

Referring to (4.45) and (4.50) completes the proof.

Proof of Theorem 4.6. Let $x^{(i)}, v^{(i)}$ be as in Proposition 4.7. By Lemma 4.4 we have $v^{(i)}=\mathfrak{p}_{\sigma_{i}}\left[\gamma_{i}, v\right]$ for all $i=0,1, \ldots, n$. We now use Proposition 4.7 and the summation by parts formula, which yields

$$
\sum_{j=0}^{n} \zeta_{j} v^{(i)}=\sum_{j=0}^{n} \zeta_{j}\left(A_{j} x^{(j)}+\sum_{i=j+1}^{n} \nu_{i} x^{(i)}\right)=x^{(0)}+\sum_{i=1}^{n} x^{(i)}\left(A_{i} \zeta_{i}+\nu_{i} B_{i-1}\right)=x,
$$

and the proof is complete.

\subsubsection{Proof of Theorem 3.3}

We can now use Theorem 4.6 to adapt the proof of Theorem 3.1 to the multi-agent case. The multi-agent system (3.11), (3.14) can still be rewritten as the equation

$$
\nabla_{t}^{2} x+A \nabla_{t} x+B \nabla_{t} s+C x_{t}+D s_{t}=0
$$

with positive constants $A, B, C, D$ given by (4.16) and $s_{t}$ as in (3.13). From the inequalities (4.4)-(4.6) we easily obtain their counterparts

$$
\begin{aligned}
& \nabla_{t} x \nabla_{t} s=\nu_{0}\left(\nabla_{t} x\right)^{2}+\sum_{i=1}^{n} \nu_{i} \nabla_{t} x \nabla_{t} s^{(i)} \geq \nu_{0}\left(\nabla_{t} x\right)^{2}+\sum_{i=1}^{n} \nu_{i}\left(\nabla_{t} s^{(i)}\right)^{2} \\
& \nabla_{t}^{2} x \nabla_{t} s=\nu_{0} \nabla_{t}^{2} x \nabla_{t} x+\sum_{i=1}^{n} \nu_{i} \nabla_{t}^{2} x \nabla_{t} s^{(i)} \geq \nu_{0} \nabla_{t}^{2} x \nabla_{t} x+\sum_{i=1}^{n} \nu_{i} \nabla_{t}^{2} s^{(i)} \nabla_{t} s^{(i)}
\end{aligned}
$$




$$
\geq \frac{\nu_{0}}{2}\left(\left(\nabla_{t} x\right)^{2}-\left(\nabla_{t-1} x\right)^{2}+\left(\nabla_{t}^{2} x\right)^{2}\right)+\sum_{i=1}^{n} \frac{\nu_{i}}{2}\left(\left(\nabla_{t} s^{(i)}\right)^{2}-\left(\nabla_{t-1} s^{(i)}\right)^{2}+\left(\nabla_{t}^{2} s^{(i)}\right)^{2}\right)
$$

where we denote $s^{(i)}:=\mathfrak{s}_{\rho_{i}}\left[\beta_{i}, x\right]$.

As in Section 4.2, we define $q_{t}$ by formula (4.18) and multiply equation (4.55) by $\nabla_{t} q$. It follows from (4.56)-(4.57) that

$$
\begin{aligned}
\nabla_{t}^{2} x \nabla_{t} q & =\left(C+\nu_{0} D\right) \nabla_{t}^{2} x \nabla_{t} x+D \sum_{i=1}^{n} \nu_{i} \nabla_{t}^{2} x \nabla_{t} s^{(i)} \geq\left(C+\nu_{0} D\right) \nabla_{t}^{2} x \nabla_{t} x+D \sum_{i=1}^{n} \nu_{i} \nabla_{t}^{2} s^{(i)} \nabla_{t} s^{(i)} \\
& \geq \frac{C+\nu_{0} D}{2}\left(\left(\nabla_{t} x\right)^{2}-\left(\nabla_{t-1} x\right)^{2}+\left(\nabla_{t}^{2} x\right)^{2}\right)+\sum_{i=1}^{n} \frac{\nu_{i} D}{2}\left(\left(\nabla_{t} s^{(i)}\right)^{2}-\left(\nabla_{t-1} s^{(i)}\right)^{2}+\left(\nabla_{t}^{2} s^{(i)}\right)^{2}\right)
\end{aligned}
$$

and

$$
\begin{aligned}
\left(A \nabla_{t} x+B \nabla_{t} s\right) \nabla_{t} q \geq & \left(A C+\nu_{0}(B C+A D)\right)\left(\nabla_{t} x\right)^{2}+B D\left(\nabla_{t} s\right)^{2} \\
& +(B C+A D) \sum_{i=1}^{n} \nu_{i}\left(\nabla_{t} s^{(i)}\right)^{2}
\end{aligned}
$$

Putting

$$
\tilde{V}_{t}^{1}:=\frac{1}{2}\left(\left(C+\nu_{0} D\right)\left(\nabla_{t} x\right)^{2}+D \sum_{i=1}^{n} \nu_{i}\left(\nabla_{t} s^{(i)}\right)^{2}+q_{t}^{2}\right)
$$

we obtain (cf. (4.20) with $\left.h_{t}=0\right)$

$$
\begin{aligned}
\tilde{V}_{t}^{1}-\tilde{V}_{t-1}^{1} & +\frac{C+\nu_{0} D}{2}\left(\nabla_{t}^{2} x\right)^{2}+\sum_{i=1}^{n} \frac{\nu_{i} D}{2}\left(\nabla_{t}^{2} s^{(i)}\right)^{2}+\left(A C+\nu_{0}(B C+A D)\right)\left(\nabla_{t} x\right)^{2} \\
& +B D\left(\nabla_{t} s\right)^{2}+(B C+A D) \sum_{i=1}^{n} \nu_{i}\left(\nabla_{t} s^{(i)}\right)^{2}+\frac{1}{2}\left(\nabla_{t} q\right)^{2} \leq 0
\end{aligned}
$$

We continue as in Section 4.2 and multiply (4.55) by $q_{t}$. Similarly to (4.22), we obtain

$$
\begin{aligned}
\nabla_{t} x q_{t}-\nabla_{t-1} x q_{t-1}+\frac{A}{2 C}\left(q_{t}^{2}-q_{t-1}^{2}\right)+\frac{1}{2} q_{t}^{2} & \leq\left(\nabla_{t} x-\nabla_{t}^{2} x\right) \nabla_{t} q+E\left(\nabla_{t} s\right)^{2}-\frac{A}{2 C}\left(\nabla_{t} q\right)^{2} \\
& \leq F\left(\frac{1}{2}\left(\nabla_{t}^{2} x\right)^{2}+\left(\nabla_{t} x\right)^{2}+\sum_{i=1}^{n} \nu_{i}\left(\nabla_{t} s^{(i)}\right)^{2}\right)
\end{aligned}
$$

with some constants $E, F>0$ depending only on $A, B, C, D$. We set

$$
\tilde{V}_{t}^{0}:=\nabla_{t} x q_{t}+\frac{A}{2 C} q_{t}^{2}
$$


and rewrite (4.58) in the form

$$
\tilde{V}_{t}^{0}-\tilde{V}_{t-1}^{0}+\frac{1}{2} q_{t}^{2} \leq F\left(\frac{1}{2}\left(\nabla_{t}^{2} x\right)^{2}+\left(\nabla_{t} x\right)^{2}+\sum_{i=1}^{n} \nu_{i}\left(\nabla_{t} s^{(i)}\right)^{2}\right),
$$

which is parallel to (4.23)-(4.24). We now define an auxiliary energy functional

$$
\tilde{W}_{t}=\tilde{V}_{t}^{1}+\lambda \tilde{V}_{t}^{0}
$$

with $\lambda>0$ such that $\lambda \tilde{V}_{t}^{0} \geq-\frac{1}{2} \tilde{V}_{t}^{1}$ and $\lambda<\min \left\{C+\nu_{0} D, A C+\nu_{0}(B C+A D), B C+A D\right\}$. We then find $\mu>0$ and $L>0$ such that for all $t \in \mathbb{N}$ we have the inequality

$$
\tilde{W}_{t}-\tilde{W}_{t-1}+\mu \tilde{W}_{t} \leq 0
$$

Therefore, the decay of $\tilde{W}_{t}$ is exponential according to the formula

$$
\tilde{W}_{t} \leq\left(\frac{1}{1+\mu}\right)^{t} \tilde{W}_{0}
$$

hence also

$$
\lim _{t \rightarrow \infty} q_{t}=0
$$

Using (3.13), we can rewrite formula (4.18) as

$$
q_{t}=\left(C+\nu_{0} D\right) x_{t}+D \sum_{i=1}^{n} \nu_{i} \mathfrak{s}_{\rho_{i}}\left[\beta_{i}, x\right]_{t}=(C+D) x_{t}-D \sum_{i=1}^{n} \nu_{i} \mathfrak{p}_{\rho_{i}}\left[\beta_{i}, x\right]_{t} .
$$

Hence, $q_{t}$ is given by a Prandtl-Ishlinskii operator of the form

$$
q_{t}=\tilde{\nu}_{0} x_{t}+\sum_{i=1}^{n} \tilde{\nu}_{i} \mathfrak{p}_{\rho_{i}}\left[\beta_{i}, x\right]_{t}
$$

with $\tilde{\nu}_{0}=C+D, \tilde{\nu}_{i}=-\nu_{i} D$ for $i=1, \ldots, n$. The hypotheses of Theorem 4.6 are satisfied for

$$
\tilde{A}_{i}:=\sum_{j=0}^{i} \tilde{\nu}_{j} \geq C+D\left(1-\sum_{j=0}^{i} \nu_{j}\right) \geq C>0 .
$$

Consequently, by Theorem 4.6, we have

$$
x_{t}=\tilde{\zeta}_{0} q_{t}+\sum_{i=1}^{n} \tilde{\zeta}_{i} \mathfrak{p}_{\sigma_{i}}\left[\gamma_{i}, q\right]_{t}
$$

with suitable constants $\tilde{\zeta}_{i}, \sigma_{i}, \gamma_{i}$. From (4.59) and from Lemma 4.3 we conclude that $x_{t}$ and $s_{t}$ are Cauchy sequences that converge to some limits $x_{\infty}, s_{\infty}$, respectively, which completes the proof of Theorem 3.3. 


\section{Conclusions}

We first replaced rational expectations about future inflation with a form of boundedly-rational aggregated 'sticky' expectation, modeled using a play operator, in a simple standard macroeconomic model. It was shown that a model with $n$ sticky representative agents has $2 n$ switching surfaces and an $n$-dimensional continuum of equilibria. We then introduced a similar form of stickiness (but motivated quite differently) into the action of the Central Bank. These conceptually quite elementary changes transformed a unique-equilibrium linear system to a PWL system with an entire continuum of equilibrium states.

By constructing Lyapunov functions and developing a technique for inverting the play operator, it was proved that in the absence of exogenous noise the continuum of equilibria is the global attractor of the system. The size of the basin of attraction of a particular equilibrium varies, typically becoming smaller towards the boundary of the set of equilibrium states.

We believe that play operators, and Prandtl-Ishlinskii operators more generally, may be a useful analytical tool for studying path-dependent economic and financial systems. The main modeling requirement is that the path-dependent characteristics are dominated by the effects of (a subset of) the previous extreme values encountered along the path. Indeed play operators have already been applied to microeconomics [20] and finance [32] and the techniques presented here may be applicable in those areas.

This paper has focused on questions of equilibria, stability and long-time dynamics but we conclude with some observations on the consequences of our analysis.

For the models studied in this paper, after sufficiently small shocks (whether exogenous or applied by policy makers) the system will indeed revert to the same equilibrium but larger shocks will move the system from the basin of attraction of one equilibrium to the basin of attraction of a different one (at the same model parameters). The path to this new equilibrium may be long with a highly unpredictable endpoint and there is no tendency to return to its pre-shocked state - the model displays true permanence.

The fact that equilibria close to the border of the equilibrium set are less stable means that the system may suddenly leave the vicinity of the equilibrium set and embark upon a long, highly-unpredictable (but bounded) excursion before returning to the neighbourhood of an equilibrium. This may happen suddenly, triggered perhaps by an unremarkable exogenous shock, after a long period of reacting proportionately to exogenous forces drifting around the equilibrium set. A numerical study of such effects will be presented elsewehere.

Acknowledgements. The authors thank Anton Cheremukhin and Luis Rios for the valuable discussion. DR was supported by NSF grant DMS-1413223, PK was supported by the European Regional Development Fund, Project No. CZ.02.1.01/0.0/0.0/16_019/0000778 and RVO: 67985840. The hospitality of the University of Texas at Dallas during PK's stay there in November 2015 is gratefully acknowledged.

\section{REFERENCES}

[1] G. Antinolfi, C. Azariadis and J.B. Bullard, Monetary policy as equilibrium selection. Rev. Federal Reserve Bank Saint Louis 89 (2007) 331-342.

[2] M. Al Janaideh, S. Rakheja and C.Y. Su, A generalized Prandtl-Ishlinskii model for characterizing the hysteresis and saturation nonlinearities of smart actuators. Smart Mater. Struct. 18 (2009) 045001.

[3] M. Arnold, N. Begun, P. Gurevich, E. Kwame, H. Lamba and D. Rachinskii, Dynamics of discrete time systems with a hysteresis stop operator. SIAM J. Appl. Dyn. Syst. 16 (2017) 91-119.

[4] V. Avrutin and I. Sushko, A gallery of bifurcation scenarios in piecewise smooth 1d maps, in Global Analysis of Dynamic Models in Economics and Finance. Springer (2013) 369-395.

[5] L.M. Ball, Hysteresis in unemployment: Old and new evidence. The National Bureau of Economic Research Working Paper 14818 (2009) 1-35.

[6] O. Blanchard and J. Wolfers, The roles of shocks and institutions in the rise of European unemployment: the aggregate evidence. Econ. J. 110 (2000) C1-C33.

[7] J. Benhabib and R.E.A. Farmer, Indeterminacy and sunspots in macroeconomics. In Vol. 1 of Handbook of Macroeconomics (1999) 387-448.

[8] A. Bick, Threshold effects of inflation on economic growth in developing countries. Econ. Lett. 108 (2010) $126-129$.

[9] M. Brokate and J. Sprekels, Hysteresis and Phase Transitions. Springer Series in Computational Mathematics. Springer Berlin Heidelberg (1996). 
[10] W.A. Branch, Sticky information and model uncertainty in survey data on inflation expectations. J. Econ. Dyn. Control 31 (2007) 245-276.

[11] C.D. Carroll, Macroeconomic expectations of households and professional forecasters. Quart. J. Econ. 118 (2003) $269-298$.

[12] G.A. Calvo, Staggered prices in a utility-maximizing framework. J. Monet. Econ. 12 (1983) 383-398.

[13] L. J. Christiano, M. Trabandt and K. Walentin, DSGE models for monetary policy analysis, in Handbook of Monetary Economics, edited by B.B. Friedman and M. Woodford. Elsevier (2010) 285-367.

[14] D. Colander, P. Howitt, A. Kirman, A. Leijonhufvud and P. Mehrling, Beyond DSGE models: toward an empirically based macroeconomics. Am. Econ. Rev. 98 (2008) 236-240.

[15] R. Curtin, Inflation expectations and empirical tests. Inflation Expect. 56 (2010) 34-61.

[16] J. Darby, R. Cross and L. Piscitelli, Hysteresis and unemployment: a preliminary investigation. Vol. 1 of The Science of Hysteresis, edited by G. Bertotti, I. Mayergoyz. Elsevier (2006) 667-699.

[17] G.W. Evans, B. McGough, Observability and equilibrium selection. Tech. rep., Mimeo, University of Oregon (2015).

[18] X. Gabaix, A sparsity-based model of bounded rationality. Quart. J. Econ. 129 (2014) 1661-1710.

[19] P.D. Grauwe, Booms and busts in economic activity: a behavioral explanation. J. Econ. Behav. Organ. 83 (2012) $484-501$.

[20] M. Göcke, Various concepts of hysteresis applied in economics. J. Econ. Surv. 16 (2002) 167-188.

[21] M. Göcke and L. Werner, Play hysteresis in supply or in demand as part of a market model. Metroeconomica 66 (2015) 339-374.

[22] J.M. Frimpong and E.F. Oteng-Abayie, When is inflation harmful? Estimating the threshold effect for Ghana. Am. J. Econ. Bus. Admin. 2 (2010) 232-239.

[23] G. Fuchs, Structural stability for dynamical economic models. J. Math. Econ. 2 (1975) 139-154.

[24] R. F. Hartl and P.M. Kort, History dependence without unstable steady state: a non-differentiable framework. J. Math. Econ. 39 (2003) 891-900.

[25] B.D. Keen, Output, inflation, and interest rates in an estimated optimizing model of monetary policy. Rev. Econ. Dyn. 12 (2009) 327-343.

[26] A. Ishlinskii, Some applications of statistical methods to describing deformations of bodies. Izv. A.N. S.S.S.R., Techn. Ser. 9 (1944) 583-590.

[27] N. Kaldor, The irrelevance of equilibrium economics. Econ. J. 82 (1972) 1237-1255.

[28] J.M. Keynes, Poverty in plenty: is the economic system self-adjusting?, The Listener, London, CWK (1934) 489-497.

[29] M.S. Khan and A.S. Senhadji, Threshold effects in the relationship between inflation and growth. IMF Staff Papers 48 (2001) $1-21$.

[30] M.A. Krasnosel'skii and A.V. Pokrovskii, Systems with Hysteresis. Springer (1989).

[31] S. Kremer, A. Bick and D. Nautz, Inflation and growth: new evidence from a dynamic panel threshold analysis. Empir. Econ. 44 (2013) 861-878.

[32] P. Krejčí, H. Lamba, S. Melnik and D. Rachinskii, Analytical solutions for a class of network dynamics with mechanical and financial applications. Phys. Rev. E 90 (2014) 032822.

[33] P. Krejčí and P. Laurençot, Hysteresis filtering in the space of bounded measurable functions. Boll. Unione Mat. Ital. 8 (2002) $755-772$.

[34] P. Krejčí, E. Kwame, H. Lamba and D. Rachinskii, A continuum of path-dependent equilibrium solutions induced by sticky expectations. Preprint arXiv:1711.08038 (2017).

[35] P. Krejčí and J. Sprekels, Elastic-ideally plastic beams and Prandtl-Ishlinskii hysteresis operators. Math. Meth. Appl. Sci. 30 (2007) 2371-2393.

[36] P. Krejčí, Hysteresis and periodic solutions of semilinear and quasilinear wave equation. Math. Z. 193 (1986) $247-264$.

[37] P. Krejčí, H. Lamba, S. Melnik and D. Rachinskii, Kurzweil integral representation of interacting Prandtl-Ishlinskii operators. Discr. Continu. Dyn. Syst. B 20 (2015) 2949-2965.

[38] P. Krejčí, H. Lamba, G.A. Monteiro and D. Rachinskii, Kurzweil integral in financial market modelling. Math. Bohem. 141 (2016) 261-286.

[39] V. Lampaert, F. Al-Bender and J. Swevers, A generalized Maxwell-slip friction model appropriate for control purposes, in 2003 IEEE International Workshop on Workload Characterization (2003) 1170-1177.

[40] G.N. Mankiw and R. Reis, Sticky information versus sticky prices: a proposal to replace the New Keynesian Phillips curve. Quart. J. Econ. 117 (2002) 1295-1328.

[41] A. Marshall, Principles of Economics. Macmillan (1890).

[42] P. Mirowski, More Heat Than Light. Cambridge University Press (1989).

[43] J.F. Muth, Rational expectations and the theory of price movements. Econometrica 29 (1961) 315-335.

[44] B.A. Maćkowiak and M. Wiederholt, Business cycle dynamics under rational inattention. Rev. Econ. Stud. 82 (2015) 15021532.

[45] G.N. Mankiw, R. Reis and J. Wolfers, Disagreement about inflation expectations. NBER Macroecon. Annu. 18 (2003) $209-248$.

[46] L. Prandtl, Ein Gedankenmodell zur kinetischen Theorie der festen Körper. J. Appl. Math. Mech. 8 (1928) 85-106.

[47] D. Rachinskii, Equivalent combinations of stops. Automat. Remote Control 59 (1998) 1370-1378.

[48] J. Robinson, History versus equilibrium. Indian Econ. J. 21 (1974) 202-213.

[49] J. Rudd and K. Whelan, Can rational expectations sticky-price models explain inflation dynamics? Am. Econ. Rev. 96 (2006) $303-320$. 
[50] I. Rychlik, A new definition of the rainflow cycle counting method. Int. J. Fatigue 9 (1987) 119-121.

[51] A.M. Sbordone, A. Tambalotti, K. Rao and K.J. Walsh, Policy analysis using DSGE models: an introduction. Econ. Policy Rev. 16 (2010) 23-43.

[52] M. Setterfield, Should economists dispense with the notion of equilibrium? J. Post Keynes. Econ. 20 (1997) $47-76$.

[53] C.A. Sims, Rational Inattention and Monetary Economics. Vol. 3 of Handbook of Monetary Economics, edited by B.M. Friedman, M. Woodford. Elsevier (2010) 155-181.

[54] C.A. Sims, Implications of rational inattention. J. Monet. Econ. 50 (2003) 665-690.

[55] N.L. Stokey, The Economics of Inaction: Stochastic Control Models with Fixed Costs. Princeton University Press (2009).

[56] J.B. Taylor, The inflation/output variability trade-off revisited. Federal Reserve Bank of Boston Conf. Ser. 38 (1994) 21-38.

[57] T. Vinayagathasan, Inflation and economic growth: a dynamic panel threshold analysis for Asian economies. J. Asian Econ. 26 (2013) 31-41. 